\title{
Solution of the master equation for Wigner's quasiprobability distribution in phase space for the Brownian motion of a particle in a double well potential
}

\author{
William T. Coffey \\ Department of Electronic and Electrical Engineering, Trinity College, Dublin 2, Ireland \\ Yuri P. Kalmykov \\ Laboratoire de Mathématiques et Physique des Systèmes, Université de Perpignan, 52, Avenue de Paul \\ Alduy, 66860 Perpignan Cedex, France \\ Serguey V. Titov \\ Department of Electronic and Electrical Engineering, Trinity College, Dublin 2, Ireland and Institute \\ of Radio Engineering and Electronics of the Russian Academy of Sciences, Vvedenskii Square 1, \\ Fryazino 141190, Russian Federation
}

(Received 9 May 2007; accepted 21 June 2007; published online 16 August 2007)

\begin{abstract}
Quantum effects in the Brownian motion of a particle in the symmetric double well potential $V(x)=a x^{2} / 2+b x^{4} / 4$ are treated using the semiclassical master equation for the time evolution of the Wigner distribution function $W(x, p, t)$ in phase space $(x, p)$. The equilibrium position autocorrelation function, dynamic susceptibility, and escape rate are evaluated via matrix continued fractions in the manner customarily used for the classical Fokker-Planck equation. The escape rate so yielded has a quantum correction depending strongly on the barrier height and is compared with that given analytically by the quantum mechanical reaction rate solution of the Kramers turnover problem. The matrix continued fraction solution substantially agrees with the analytic solution. Moreover, the low-frequency part of the spectrum associated with noise assisted Kramers transitions across the potential barrier may be accurately described by a single Lorentzian with characteristic frequency given by the quantum mechanical reaction rate. (C) 2007 American Institute of Physics.
\end{abstract} [DOI: $10.1063 / 1.2759486]$

\section{INTRODUCTION}

Dissipation and fluctuation of an assembly of particles in a potential under the influence of a heat bath is very often modeled $^{1,2}$ by the Brownian motion which is a particular Stosszahlansatz (essentially collisions are frequent but weak) for the Boltzmann equation describing the time evolution of the single particle distribution function in phase space. The Brownian motion is treated either by the Langevin equation, which is the equation of motion of the canonical variables (position $x$ and momentum $p$ ) specifying a particle in phase space supplemented by stochastic terms representing all the other bath degrees of freedom or by the equivalent FokkerPlanck equation describing the evolution of the probability density function of the realizations of the canonical variables in phase space, which in the present context are of course random variables. A theory of dissipation so based may, however, fail particularly at low temperatures because quantum effects are excluded. Examples of these are quantum fluctuations in nanoscale and biological systems, ${ }^{3}$ flux quantum transitions in a superconducting quantum interference device, ${ }^{4}$ etc. Hence it is desirable to develop a theory of quantum Brownian motion preferably via a quantum master equation analogous to the classical Fokker-Planck equation which will allow one to study ${ }^{5}$ the semiclassical limit and so determine the role played by quantum effects in the Brownian motion. Ideally, such an equation would allow dynamical parameters such as escape rates, correlation times, susceptibilities, etc., to be calculated in a manner similar to that of the Fokker-Planck equation. The concept of a diffusion equation in phase space in the context of quantum mechanics encounters difficulties, however, because the uncertainty principle $^{6,7}$ immediately precludes one from defining a probability that the particle has a particular position and a particular momentum as in classical mechanics. In spite of this, quasiprobability density functions ${ }^{6-12}$ in phase space, such as the Wigner distribution function, ${ }^{6}$ which essentially represents quantum mechanics as a statistical theory on classical phase space, are very useful in quantum statistical mechanics. The first of these quasiprobability distributions was introduced by Wigner ${ }^{6}$ in 1932 in order to study in semiclassical fashion quantum corrections to the Maxwell-Boltzmann distribution of classical statistical mechanics, which inter alia elucidated the role played by tunneling effects at high temperatures in reaction rate theory. The Wigner distribution function was meant to be a reformulation, using the concept of a quasiprobability distribution in phase space, of Schrödinger's wave mechanics which describes quantum states of functions in configuration space. Thus the Wigner function is of particular use in the statistical mechanics of closed systems as it effectively allows one to consider quantum corrections to the classical distribution function up to any desired order of Planck's constant $\hbar$. Indeed, the Wigner distribution function is central to the purpose (namely, quan- 
tum effects in the double well potential) of the present paper as it allows one via a quantum master equation to consider quantum corrections up to $O\left(\hbar^{2 r}\right)$ ( $\hbar$ is Planck's constant) to the classical distribution function using methods familiar in the solution of the Fokker-Planck equation.

We continue by remarking that in applications to quantum Brownian motion in an external potential $V(x)$, the phase space formalism has been applied to a harmonic oscillator by Agarwal $^{13}$ and others (see, e.g., Refs. 14-19, and references cited therein). Furthermore, some other aspects of the quantum Brownian motion in anharmonic potentials have also been treated in the literature (see, e.g., Refs. 20-26). In particular, García-Palacios and Zueco ${ }^{23}$ have indicated how Risken's ${ }^{1}$ efficient method of solving the classical FokkerPlanck equation by matrix continued fractions ${ }^{1}$ may be adapted to the solution of the master equation for the quantum Brownian motion in an anharmonic potential. Inspired by these ideas we have recently proposed a quantum master equation for the Brownian motion of a particle in a general potential $V(x) .{ }^{24,25}$ Specifically we have demonstrated how the Wigner stationary distribution for closed systems can be used to formally establish a semiclassical master equation allowing one to study the quantum-classical correspondence. Furthermore we have shown in Ref. 26 how this master equation can be solved in the case of quantum Brownian motion in a periodic cosine potential. As a second example of solving the master equation for the Wigner quasiprobability distribution in a particular problem, we now study the Brownian motion of a particle in a double well potential, viz.,

$$
V(x)=a x^{2} / 2+b x^{4} / 4,
$$

where $a$ and $b$ are constants. We remark that diffusion in the potential given by Eq. (1) is almost invariably used to model noise driven motion in bistable physical and chemical systems. Examples are diverse subjects such as simple isometrization processes, ${ }^{27-31}$ chemical reaction rate theory, ${ }^{32-40}$ bistable nonlinear oscillators, ${ }^{41-43}$ second order phase transitions, ${ }^{44}$ nuclear fission and fusion, ${ }^{45,46}$ stochastic resonance, ${ }^{47,48}$ etc.

The dissipative barrier crossing process we have mentioned is characterized by the (Kramers) escape rate $\Gamma$ which may be calculated in closed form using ingenious asymptotic methods devised by Kramers ${ }^{6}$ to approximately solve the Fokker-Planck equation governing the Brownian motion in the potential in the limits of very high and low dissipations to the heat bath. These limits (which constitute a benchmark providing a check on the accuracy of numerical solutions of both Fokker-Planck and quantum master equations) are defined via a loss parameter $\Delta$ introduced by Kramers. ${ }^{6}$ The loss parameter $\Delta$ is the ratio of the energy loss per cycle $\gamma S$ of the almost periodic motion very near or at the top of a well of a (potentially escaping) particle of mass $m$ to the mean thermal energy $k T$, where $\gamma=\zeta / m$ is the damping parameter, $\zeta \dot{x}=\gamma p$ is the viscous drag on the Brownian particle arising from the heat bath, $S$ is the action of a particle in the well along the saddle point energy trajectory, and $p$ is the momentum of the particle. The saddle point energy comprises the energy trajectory on which the particle may or may not escape, i.e., it defines the separatrix between the bounded motion in the well and the unbounded motion outside. High dissipation corresponds to $\Delta \gg 1$, vice versa $\Delta$ $\ll 1$ corresponds to low dissipation. Kramers ${ }^{32}$ was, however, unable to find asymptotic solutions for $\Gamma$ in the turnover region where the energy loss is of the order of the thermal energy, i.e., $\Delta \approx 1$. The Kramers idea was later elaborated upon by Mel'nikov ${ }^{49}$ and Mel'nikov and Meshkov. ${ }^{50}$ They proposed a universal formula (that is valid for all values of the dissipation $\Delta$ ) for $\Gamma$, i.e., they solved the so called Kramers turnover problem. This was accomplished in terms of the product of an integral called the depopulation factor involving $\Delta$ and the Kramers high damping rate which as it's lower limit of applicability contains the transition state theory result (which unlike the Kramers treatment ignores the disturbance to the Maxwell-Boltzmann distribution in the wells due to escape of particles over the barrier), and the particular case is called intermediate damping. The depopulation factor corrects the intermediate-to-high damping (IHD) asymptote, namely, $\Gamma_{\mathrm{IHD}}$ (the prefactor of which is essentially inversely proportional to the friction coefficient for $\Delta \gg 1$ ) in the region $\Delta=1$ so that it joins smoothly onto the very low damping asymptote (VLD) $\Gamma_{\mathrm{VLD}}$ (which is characterized by a prefactor proportional to the friction $\gamma$ ). The different frictional behavior of the prefactor for $\Delta \gg 1$ and $\Delta \ll 1$ has given rise to the term Kramers turnover region characterizing $\Delta \approx 1$. Later Grabert ${ }^{51}$ and Pollak et al. ${ }^{52}$ presented a complete solution of the classical Kramers turnover problem deriving Mel'nikov's turnover formula for $\Gamma$ without his ad hoc interpolation between the weak and strong damping regimes. We remark that the theory of Pollak et al. ${ }^{52}$ also applies to an arbitrary memory friction and not just in the white noise limit. Furthermore, Mel'nikov ${ }^{49}$ and Rips and Pollak ${ }^{53}$ have extended the solution of the Kramers turnover problem to account for quantum tunneling in a semiclassical way. The asymptotic solutions of Kramers and their extension to all values of the damping given by Mel'nikov and Meshkov and others constitute important analytic benchmarks. In the classical case, they have been exhaustively verified for the double well potential by calculating the smallest nonvanishing eigenvalue of the Fokker-Planck equation (known in this instance as the Klein-Kramers equation) for the phase space distribution function $W(x, p, t)$ by continued fraction methods. ${ }^{54-57}$ The quantum Mel'nikov universal formula for the double well potential has been tested, on the other hand, by a comparison with numerical simulation results for quantum rate constants by Topaler and Makri ${ }^{58,59}$ (by using the path integral approach) and Barik et al. ${ }^{60}$ (by solving numerically the underlying quantum Langevin equation), the latter being based on the Wigner phase space distribution function.

Proceeding, we now consider the semiclassical master equation for the quantum Brownian dynamics in the double well potential. Specifically, we shall apply the matrix continued fraction method of Voigtlaender and Risken ${ }^{54}$ and Coffey et al. ${ }^{56}$ (developed for the solution of the corresponding classical problem) to ascertain how quantum effects modify the behavior of the quantum equilibrium position correlation function $C(t)=\beta^{-1}\left\langle\int_{0}^{\beta} \hat{x}(-i \lambda \hbar) \hat{x}(t) d \lambda\right\rangle_{0}$, its spectrum, and correlation time, which essentially yields the escape rate. Here 
the symbol \langle\rangle$_{0}$ denotes the equilibrium ensemble averages and $\beta=1 /(k T)$. This calculation will illustrate how to evaluate observables in the familiar classical manner. Moreover, the continued fraction results for the damping dependence of the quantum escape rate $\Gamma$ will be compared with those yielded by the Mel'nikov universal equation for $\Gamma$ so that the range of validity of the semiclassical master equation approach may be ascertained.

\section{MASTER EQUATION IN PHASE SPACE}

Wigner ${ }^{6}$ showed that quantum mechanics can be reformulated using a phase space $(x, p)$ quasiprobability distribution function

$$
W(x, p, t)=\frac{1}{2 \pi \hbar} \int_{-\infty}^{\infty} \rho\left(x+\frac{1}{2} y, x-\frac{1}{2} y\right) e^{-i p y / \hbar} d y,
$$

where $\rho\left(x, x^{\prime}\right)=\left\langle x|\hat{\rho}| x^{\prime}\right\rangle$ is the density matrix. Thus the Wigner distribution function establishes a connection between the density matrix and a quasiprobability distribution in classical phase space. Moreover, one can calculate all quantum mechanical averages by pure $c$-number procedures, that is, by evaluation of averages just as in classical statistical mechanics. A detailed discussion of Wigner distribution functions is given in Refs. 8-12.

Here the semiclassical master equation for the translational Brownian motion of a particle in a potential $V(x)$ based on Wigner's phase space formulation can be derived by proceeding to the high temperature limit, where the quantum parameter $\hbar^{2} \beta^{2} / 24 m \ll 1$, and using the approximation of frequency independent damping. The resulting equation to order $\hbar^{2}$ is 24,25

$$
\begin{aligned}
& \frac{\partial}{\partial t} W+\frac{p}{m} \frac{\partial W}{\partial x}-\frac{\partial V}{\partial x} \frac{\partial W}{\partial p}+\frac{\hbar^{2}}{24} \frac{\partial^{3} V}{\partial x^{3}} \frac{\partial^{3} W}{\partial p^{3}}+\cdots \\
& =\gamma \frac{\partial}{\partial p}\left[p W+\frac{m}{\beta}\left\{1+\frac{\hbar^{2} \beta^{2}}{12 m} \frac{\partial^{2} V}{\partial x^{2}}+\cdots\right\} \frac{\partial W}{\partial p}\right],
\end{aligned}
$$

where $m$ is the mass of the particle, $\gamma$ is a friction parameter measuring the strength of the coupling to the heat bath. The left hand side of Eq. (2) is the quantum analog of the classical Liouville equation for the closed system while the right hand side accounts for effects due to the coupling to the bath, i.e., dissipation and fluctuations being the analog of the collision kernel (stosszahlansatz) in kinetic theory. Equation (2) is a partial differential equation for the evolution of the quasiprobability distribution $W$ in phase space akin to the Fokker-Planck equation immediately suggesting how the familiar powerful computational techniques developed for that equation $^{1}$ may be extended to the quantum domain as we have previously demonstrated for a cosine periodic potential. $^{25}$ The master equation [Eq. (2)] is written down explicitly up to $O\left(\hbar^{2}\right)$. Higher order quantum correction terms to the master Eq. (2), may be calculated in like manner. $^{24,25}$ For example, the explicit form of the master equation up to $O\left(\hbar^{4}\right)$ is given in Refs. 24 and 25. That equation can be given, in principle, to any desired degree $r$ of $\hbar^{2 r}$.
We recall in passing that the corresponding master equation for the quantum Brownian oscillator in the weak coupling limit, $\beta \hbar \gamma \ll 1$, originally studied by Agarwal ${ }^{13}$ is $^{25}$

$$
\frac{\partial W}{\partial t}+\frac{p}{m} \frac{\partial W}{\partial x}-m \omega_{0}^{2} x \frac{\partial W}{\partial p}=\gamma \frac{\partial}{\partial p}\left[p W+\left\langle p^{2}\right\rangle_{0} \frac{\partial W}{\partial p}\right],
$$

where $\omega_{0}$ is the oscillator frequency and $\left\langle p^{2}\right\rangle_{0}$ $=\left(m \hbar \omega_{0} / 2\right) \operatorname{coth}\left(\beta \hbar \omega_{0} / 2\right)$. Here, Eq. (3) has the same mathematical form as the Fokker-Planck equation for a classical Brownian oscillator, ${ }^{1}$ however, the diffusion coefficient $D_{p p}$ $=\gamma\left\langle p^{2}\right\rangle_{0}$ is altered so as to include the quantum effects. This simple result essentially arises because the dynamical equation for the Wigner function for a quadratic Hamiltonian $\hat{H}$ $=\hat{p}^{2} / 2 m+m \omega_{0}^{2} \hat{x}^{2} / 2$ in the absence of dissipation $(\gamma=0)$ coincides with the corresponding classical Liouville equation. ${ }^{17}$

\section{SOLUTION OF THE MASTER EQUATION}

In order to solve the master equation for the double well potential given by Eq. (1) (which is accomplished by perturbation theory in $\hbar^{2}$ with the perturbation expansion truncated at the terms linear in $\hbar^{2}$ ) we begin by introducing dimensionless variables as in the classical model ${ }^{56}$

$$
\begin{aligned}
& x^{\prime}=\frac{x}{\sqrt{\left\langle x^{2}\right\rangle_{0}^{\mathrm{cl}}}}, \quad p^{\prime}=\frac{\eta p}{m \sqrt{\left\langle x^{2}\right\rangle_{0}^{\mathrm{cl}}}}, \\
& t^{\prime}=t / \eta, \quad \Lambda=\hbar^{2} \beta^{2} /\left(48 \eta^{2}\right), \\
& \gamma^{\prime}=\eta \gamma, \quad V\left(x^{\prime}\right)=A x^{\prime 2}+B x^{\prime 4}, \quad A=\beta a\left\langle x^{2}\right\rangle_{0}^{\mathrm{cl}} / 2, \\
& B=\beta b\left(\left\langle x^{2}\right\rangle_{0}^{\mathrm{cl}}\right)^{2} / 4,
\end{aligned}
$$

where $\eta=\sqrt{\beta m\left\langle x^{2}\right\rangle_{0}^{\mathrm{cl}} / 2}$ is a characteristic time and $\left\langle x^{2}\right\rangle_{0}^{\mathrm{cl}}$ is the classical value $(\hbar \rightarrow 0)$ of the mean squared displacement. For $A>0$ and $B>0$, the potential $V\left(x^{\prime}\right)$ has only one minimum. For $A<0$ and $B>0$ (which is the case of interest, i.e., distinct double wells), the potential $V\left(x^{\prime}\right)$ has two minima separated by a maximum at $x^{\prime}=0$ with potential barrier $\Delta V=Q=A^{2} / 4 B$. The normalization condition $\left\langle x^{\prime 2}\right\rangle_{0}^{\mathrm{cl}}=1$ implies that the constants $A$ and $B$ are not independent and are related via $^{2,55,56}$

$$
B(Q)=\frac{1}{8} \frac{D_{-3 / 2}^{2}(\operatorname{sgn}(A) \sqrt{2 Q})}{D_{-1 / 2}^{2}(\operatorname{sgn}(A) \sqrt{2 Q})},
$$

where $D_{v}(z)$ is Whitaker's parabolic cylinder function of or$\operatorname{der} v .^{61}$ For $A<0$ and $Q \gg 1, B \approx Q$ due to asymptotic properties of $D_{v}(z){ }^{61}$ Thus Eq. (2) becomes

$$
\begin{aligned}
\frac{\partial}{\partial t^{\prime}} W & +p^{\prime} \frac{\partial W}{\partial x^{\prime}}-\frac{1}{2} \frac{\partial W}{\partial p^{\prime}} \frac{\partial V}{\partial x^{\prime}}+\frac{\Lambda}{4} \frac{\partial^{3} W}{\partial p^{\prime 3}} \frac{\partial^{3} V}{\partial x^{\prime 3}} \\
& =\gamma^{\prime} \frac{\partial}{\partial p^{\prime}}\left\{p^{\prime} W+\left[\frac{1}{2}+\Lambda \frac{\partial^{2} V}{\partial x^{\prime 2}}\right] \frac{\partial W}{\partial p^{\prime}}\right\}+O\left(\Lambda^{2}\right) .
\end{aligned}
$$

The stationary solution of Eq. (5) is the equilibrium Wigner distribution function $W_{\mathrm{st}}^{0}\left(x^{\prime}, p^{\prime}\right)$ restricted to the term linear in the quantum parameter $\Lambda$ and given by ${ }^{6,24}$ 


$$
\begin{aligned}
W_{\mathrm{st}}^{0}\left(x^{\prime}, p^{\prime}\right)= & \frac{e^{-p^{\prime 2}-V\left(x^{\prime}\right)}}{\sqrt{\pi} Z}\left\{1+\Lambda\left[\left(2 p^{\prime 2}-3\right) \frac{\partial^{2} V\left(x^{\prime}\right)}{\partial x^{\prime 2}}\right.\right. \\
& \left.\left.+\left(\frac{\partial V\left(x^{\prime}\right)}{\partial x^{\prime}}\right)^{2}\right]+O\left(\Lambda^{2}\right)\right\}
\end{aligned}
$$

where $Z$ is the partition function in accordance with our first order perturbation ansatz given by

$$
Z=\int_{-\infty}^{\infty} \int_{-\infty}^{\infty} W_{\mathrm{st}}^{0}\left(x^{\prime}, p^{\prime}\right) d x^{\prime} d p^{\prime}=Z_{\mathrm{cl}}+\Lambda Z_{1}+O\left(\Lambda^{2}\right),
$$

where $Z_{\mathrm{cl}}=\int_{-\infty}^{\infty} e^{-V\left(x^{\prime}\right)} d x^{\prime}$ is the classical partition function in configuration space and $Z_{1}=\int_{-\infty}^{\infty}\left[V^{\prime}\left(x^{\prime}\right)^{2}-2 V^{\prime \prime}\left(x^{\prime}\right)\right] e^{-V\left(x^{\prime}\right)} d x^{\prime}$.

According to linear response theory, ${ }^{62}$ in order to calculate a position correlation function $C(t)$, one must evaluate the decay transient of the system of Brownian particles following instantaneous switch-off of an external field of small magnitude $\varepsilon$. Thus when the field is suddenly switched off at time $t=0$ we shall be interested in the relaxation of a system starting from an equilibrium state I with the potential $V\left(x^{\prime}\right)$ $-\varepsilon x^{\prime}$ and the distribution function $W_{\mathrm{st}}^{\varepsilon}(t \leqslant 0)$ to a new equilibrium state II with the potential $V\left(x^{\prime}\right)$ and the distribution function $W_{\mathrm{st}}^{0}(t \rightarrow \infty)$ given by Eq. (6). In linear response the distribution function $W_{\mathrm{st}}^{\varepsilon}$ is given by

$$
W_{\mathrm{st}}^{\varepsilon}\left(x^{\prime}, p^{\prime}\right)=W_{\mathrm{st}}^{0}\left(x^{\prime}, p^{\prime}\right)+\varepsilon W_{1}^{0}\left(x^{\prime}, p^{\prime}\right)+O\left(\varepsilon^{2}\right),
$$

where

$$
\begin{aligned}
W_{1}^{0}\left(x^{\prime}, p^{\prime}\right)= & x^{\prime} W_{\mathrm{st}}^{0}\left(x^{\prime}, p^{\prime}\right)-2 \Lambda \frac{e^{-p^{\prime 2}-V\left(x^{\prime}\right)}}{\sqrt{\pi} Z_{\mathrm{cl}}} \frac{\partial}{\partial x^{\prime}} V\left(x^{\prime}\right) \\
& +O\left(\Lambda^{2}\right) .
\end{aligned}
$$

Note that the transient response so formulated is truly linear because the change in amplitude $\varepsilon$ of the external field is assumed to be very small, $\varepsilon \rightarrow 0$. Hence we seek a general solution of Eq. (5) in the form

$$
W\left(x^{\prime}, p^{\prime}, t^{\prime}\right)=W_{\mathrm{st}}^{0}\left(x^{\prime}, p^{\prime}\right)+\varepsilon W_{1}\left(x^{\prime}, p^{\prime}, t^{\prime}\right)+O\left(\varepsilon^{2}\right),
$$

where $W_{1}\left(x^{\prime}, p^{\prime}, t^{\prime}\right)$ can be represented as the Fourier series just as the classical case ${ }^{54,56}$

$$
\begin{aligned}
& W_{1}\left(x^{\prime}, p^{\prime}, t^{\prime}\right) \\
& \quad=\kappa e^{-p^{\prime 2}-\left[\kappa^{2} x^{\prime 2}+V\left(x^{\prime}\right)\right] / 2} \sum_{n=0}^{\infty} \sum_{q=0}^{\infty} \frac{H_{q}\left(\kappa x^{\prime}\right) H_{n}\left(p^{\prime}\right)}{\pi \sqrt{2^{n+q} n ! q !}} c_{n, q}\left(t^{\prime}\right),
\end{aligned}
$$

where $H_{n}(z)$ are the orthogonal Hermite polynomials, ${ }^{61} \kappa$ $=\alpha B^{1 / 4}$, and $\alpha$ is a scaling factor chosen to ensure optimum convergence of the continued fractions involved as suggested by Voigtlaender and Risken ${ }^{54}$ (all results for the observables are independent of $\alpha$ ). The initial condition for $W\left(x^{\prime}, p^{\prime}, t^{\prime}\right)$ at $t^{\prime}=0$ is $W\left(x^{\prime}, p^{\prime}, 0\right)=W_{\mathrm{st}}^{\varepsilon}\left(x^{\prime}, p^{\prime}\right)$ which in linear response becomes

$$
W_{1}\left(x^{\prime}, p^{\prime}, 0\right)=W_{1}^{0}\left(x^{\prime}, p^{\prime}\right) .
$$

By substituting Eq. (9) into Eq. (5) and noting that ${ }^{61}$

$$
\frac{d}{d z} H_{n}(z)=2 n H_{n-1}(z), \quad H_{n+1}(z)=2 z H_{n}(z)-2 n H_{n-1}(z),
$$

we have the differential recurrence relations for the Fourier coefficients $c_{n, q}(t)$,

$$
\begin{aligned}
& \frac{d}{d t^{\prime}} c_{n, q}\left(t^{\prime}\right)+\gamma^{\prime}\left[n c_{n, q}\left(t^{\prime}\right)-\Lambda \sqrt{n(n-1)}\left(h_{q-2} c_{n-2, q-2}\right.\right. \\
& \left.\left.\quad+g_{q} c_{n-2, q}+h_{q} c_{n-2, q+2}\right)\right]=\sqrt{n+1}\left[e_{q} c_{n+1, q+3}\left(t^{\prime}\right)\right. \\
& \left.\quad+d_{q}^{-} c_{n+1, q+1}\left(t^{\prime}\right)+d_{q-1}^{+} c_{n+1, q-1}\left(t^{\prime}\right)+e_{q-3} c_{n+1, q-3}\left(t^{\prime}\right)\right] \\
& \quad-\sqrt{n}\left[e_{q} c_{n-1, q+3}\left(t^{\prime}\right)+d_{q}^{+} c_{n-1, q+1}\left(t^{\prime}\right)+d_{q-1}^{-} c_{n-1, q-1}\left(t^{\prime}\right)\right. \\
& \left.\quad+e_{q-3} c_{n-1, q-3}\left(t^{\prime}\right)\right]+\Lambda \sqrt{n(n-1)(n-2)}\left[f_{q-1} c_{n-3, q-1}\right. \\
& \left.\quad+f_{q} c_{n-3, q+1}\right]+O\left(\Lambda^{2}\right),
\end{aligned}
$$

where all the coefficients $d_{p}^{ \pm}, e_{q}, f_{q}, g_{q}$, and $h_{q}$ are defined in Appendix A. Now Eq. (12) reduces by first order perturbation treatment in $\Lambda$ to a matrix three-term differential recurrence relation forced by the quantum term. Thus by invoking the familiar general matrix continued fraction method for solving classical recurrence relations generated by the Fokker-Planck equation, ${ }^{1,2}$ we have in a similar manner the solution of the quantum differential recurrence equation [Eq. (12)] (details of this solution are given in Appendix A).

\section{CALCULATION OF OBSERVABLES}

We recall now that the expectation value of a quantum operator $\hat{Q}$ may be calculated using the Wigner function $W(x, p, t)$ from the corresponding classical variable (Weyl symbol) $Q(x, p)$ as $^{7}$

$$
\langle\hat{Q}\rangle(t)=\int W(x, p, t) Q(x, p) d x d p .
$$

Noting that $x^{\prime}$ corresponds to the operator $\hat{x}^{\prime},{ }^{7}$ we have the averaged displacement $\left\langle\hat{x}^{\prime}\right\rangle\left(t^{\prime}\right)$ as

$$
\begin{aligned}
\left\langle\hat{x}^{\prime}\right\rangle\left(t^{\prime}\right) & =\int_{-\infty}^{\infty} \int_{-\infty}^{\infty} x^{\prime} W\left(x^{\prime}, p^{\prime}, t^{\prime}\right) d x^{\prime} d p^{\prime} \\
& =\varepsilon \int_{-\infty}^{\infty} \int_{-\infty}^{\infty} x^{\prime} W_{1}\left(x^{\prime}, p^{\prime}, t^{\prime}\right) d x^{\prime} d p^{\prime} .
\end{aligned}
$$

By using the orthogonality properties of the Hermite polynomials, we have from Eqs. (10) and (13) $\left\langle\hat{x}^{\prime}\right\rangle\left(t^{\prime}\right)$ in terms of the Fourier coefficients $c_{2 q-1}(t)$

$$
\left\langle\hat{x}^{\prime}\right\rangle\left(t^{\prime}\right)=\varepsilon \frac{\alpha Z_{\mathrm{cl}} B^{1 / 4}}{\sqrt{\pi}} \sum_{q=1}^{\infty} c_{0,2 q-1}(0) c_{0,2 q-1}(t) .
$$

According to linear response theory, ${ }^{62}\left\langle\hat{x}^{\prime}\right\rangle\left(t^{\prime}\right)$ is related to the linear response after-effect function $C(t)$ $=\beta^{-1}\left\langle\int_{0}^{\beta} \hat{x}^{\prime}(-i \lambda \hbar) \hat{x}^{\prime}(t) d \lambda\right\rangle_{0}$ via

$$
\left\langle\hat{x}^{\prime}\right\rangle\left(t^{\prime}\right)=\varepsilon C(t) .
$$

This may be verified in the quantum case by independently calculating both the after-effect function and the autocorrelation function from the Fourier coefficients. The one-sided Fourier transform $\widetilde{C}(\omega)=\int_{0}^{\infty} C(t) e^{-i \omega t} d t$, i.e., the spectrum of 
the equilibrium correlation function $C(t)$ is related to the dynamic susceptibility $\chi(\omega)=\chi^{\prime}(\omega)-i \chi^{\prime \prime}(\omega)$ via

$$
\chi(\omega)=C(0)-i \omega \tilde{C}(\omega) .
$$

One may also determine the correlation time $T_{c}$, which is a global characteristic of the relaxation process involved and is defined as the area under the curve of $C(t) / C(0)$, because $^{2}$

$$
T_{c}=\frac{1}{C(0)} \int_{0}^{\infty} C(t) d t=\frac{\widetilde{C}(0)}{C(0)} .
$$

In the high barrier limit $(Q \gg 1)$, the correlation time $T_{c}$ closely approximates the inverse Kramers escape rate, i.e., the greatest relaxation time for the double well potential. ${ }^{1}$

\section{MEL'NIKOV'S UNIVERSAL FORMULA FOR THE ESCAPE RATE}

We have mentioned that Mel'nikov ${ }^{49}$ extended his solution of the classical Kramers turnover problem to include quantum effects in a semiclassical way. $\mathrm{He}$ accomplished this by simply inserting the quantum mechanical transmission factor for a parabolic barrier (which assumes that tunneling transitions near the top of the barrier predominate) into the classical integral equation for the energy distribution function yielded by the Wiener-Hopf method based on the Green function of an energy/action diffusion representation of the Klein-Kramers equation in the Kramers turnover region where the loss parameter $\Delta \sim 1$. In the approximation of Ohmic damping, he derived a universal formula valid for all values of damping for the quantum rate $\Gamma_{M}$ above the crossover temperature between tunneling and thermal activation, namely,

$$
\Gamma_{M}=Y \Gamma_{\mathrm{IHD}} .
$$

Here $Y$ is the quantum depopulation factor, $\Gamma_{I H D}$ is the quantum escape rate for the double well potential in the IHD region where $\gamma^{\prime} \geqslant 1$ and $^{23}$

$$
\Gamma_{\mathrm{IHD}}=\Xi \Gamma_{\mathrm{IHD}}^{\mathrm{cl}} \text {. }
$$

Here $\Gamma_{\mathrm{IHD}}^{\mathrm{cl}}$ is the classical IHD escape rate for the double well potential given by ${ }^{56}$

$$
\Gamma_{\mathrm{IHD}}^{\mathrm{cl}}=\frac{\Omega \omega_{c}}{\pi \omega_{a}} e^{-\beta \Delta V}
$$

where $\beta \Delta V=Q$ is the normalized barrier height, $\omega_{a}$ $=\sqrt{2} \eta^{-1}(Q B)^{1 / 4}$ and $\omega_{c}=2 \eta^{-1}(Q B)^{1 / 4}$ are, respectively, the well and barrier angular frequencies, $\Omega=\eta^{-1}\left(\sqrt{\gamma^{\prime 2} / 4+\eta^{2} \omega_{a}^{2}}\right.$ $\left.-\gamma^{\prime} / 2\right)$ is the eigenvalue associated with the unstable barrier crossing mode in the notation of the present paper, and the quantum correction factor $\Xi$ is

$$
\Xi=\frac{\omega_{c} \sinh \left(\hbar \beta \omega_{a} / 2\right)}{\omega_{a} \sin \left(\hbar \beta \omega_{c} / 2\right)} .
$$

The quantum depopulation factor $Y$ for a symmetric double well potential can be written as ${ }^{49}$

$$
\Upsilon(\Delta, y)=A^{\prime 2}(\Delta, y) / A^{\prime}(2 \Delta, y) .
$$

Here $A^{\prime}$ is the quantum depopulation factor for a single well, $y=2 \sqrt{6 \Lambda} \sqrt{Q B}$ is a dimensionless parameter, depending on the ratio of the quantum parameter $\Lambda$ to the barrier height parameter, and $\Delta$ is the loss parameter as defined in Sec. I, namely,

$$
\Delta=\beta \gamma^{\prime} S / \eta,
$$

where $S=\oint_{\text {well }} \sqrt{-2 m V(x)} d x$ is the action associated with the path along the top of barrier given by

$$
S=2 \int_{0}^{x_{1}} \sqrt{-2 m V(x)} d x=\frac{8 \sqrt{2} \eta\left(Q^{3} / B\right)^{1 / 4}}{3 \beta}
$$

$\left[x_{1}=\sqrt{-2 a / b}\right.$ is one of the roots of the equation $\left.V(x)=0\right]$. On this path, a particle starts with zero velocity at the top of the barrier and, having descended into the well, returns again to the top of the barrier. For $Q \gg 1, S \sim(8 \eta / 3 \beta) \sqrt{2 Q}$. The quantum depopulation factor for a single well $A^{\prime}(\Delta, y)$ is given by $^{49}$

$$
A^{\prime}(\Delta, y)=\exp \left\{\frac{y \sin y}{\pi} \int_{-\infty}^{\infty} \frac{\ln \left[1-e^{-\Delta R(\lambda, y)}\right] d \lambda}{\cosh (2 y \lambda)-\cos y}\right\} .
$$

The expression for $R(\lambda, y)$ depends on the precise form of the well potential ${ }^{49}$ and has been given explicitly in Ref. 49 for cubic and periodic potentials. The derivation of this function for the double well potential is given in Appendix B [Eq. (B5)]. Thus one may evaluate the escape rate [from Eqs. (18)-(25) and (B5)] which may then be compared with the semiclassical solution obtained from Eq. (12) using matrix continued fractions. It should be noted that the universal Eq. (18) for the escape rate can be used only for high barriers (say $Q \geqslant 3$ ) with, however, no limitation on the quantum parameter $\Lambda$.

\section{RESULTS AND DISCUSSION}

In Figs. 1 and 2 we show the spectrum of $\operatorname{Im}[\widetilde{C}(\omega)]$ and $\operatorname{Re}[\tilde{C}(\omega)]$ as calculated from the series of Fourier coefficients Eq. (14) by the matrix continued fraction method for barrier height parameters $Q=5$ and 10 and various values of $\gamma^{\prime}=1,10$, and 100. The low-frequency part of the spectra $\widetilde{C}(\omega)$ is due to the slow overbarrier relaxation of the particles in the double well potential. Just as in the classical case, ${ }^{56}$ this low-frequency part may by approximated by a simple Lorentzian,

$$
\widetilde{C}(\omega) \approx \frac{1}{\Gamma_{M}+i \omega},
$$

where $\Gamma_{M}$ is the escape rate rendered by Melnikov's universal quantum rate, Eq. (18). It is apparent from Figs. 1 and 2 that the simple equation [Eq. (26)] accurately describes the quantum effects in the relaxation phenomenon at low frequencies $\left(\omega \leqslant \Gamma_{M}\right)$. It is also apparent from Fig. 1 particularly in the high barrier case that tunnelling near the top of the barrier increases the frequency of the maximum $\omega_{R}$ $=\Gamma_{M}$ of the low-frequency peak in $\operatorname{Im}[\widetilde{C}(\omega)]$ and decreases 

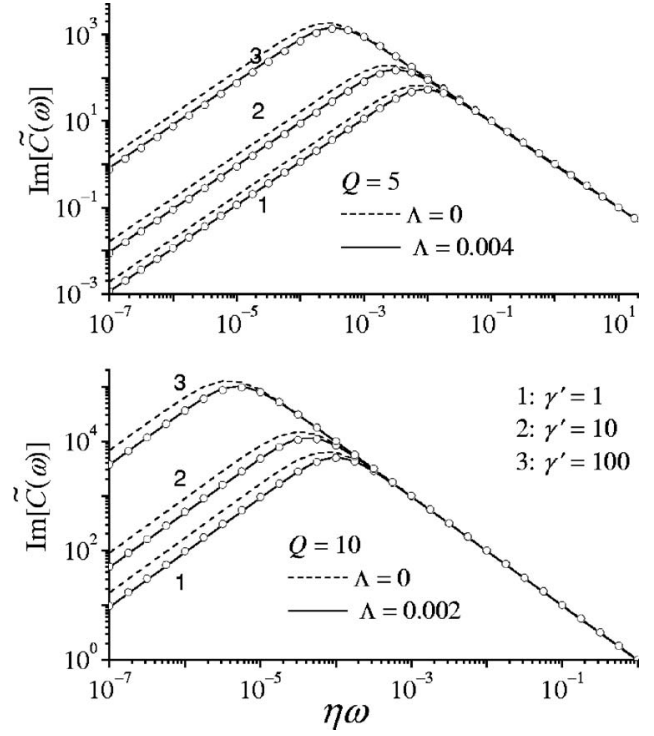

FIG. 1. The imaginary part of the spectrum $\widetilde{C}(\omega)$ vs the normalized frequency $\eta \omega$ for various values of the damping parameter $\gamma^{\prime}$, quantum parameter $\Lambda=0$ (classical limit, dashed lines) and $\Lambda=0.004$ (solid lines), and barrier height $Q=5$ and $Q=10$. Solid and dashed lines are the continued fraction solution [Eq. (14) and Appendix A]. Open circles are the Lorentzian spectra [Eq. (26)] for $\Lambda=0.004$.

the low-frequency side of $\operatorname{Im}[\widetilde{C}(\omega)]$; a phenomenon which can be ascribed to effective lowering of the potential barrier due to the tunneling which increases the escape rate. Furthermore, the quantum effects decrease as the frequency increases above the peak frequency, where barrier crossing is no longer the dominant relaxation process. As far as the dependence of the low-frequency part of the spectrum is concerned, the frequency $\omega_{R}$ decreases as the damping parameter $\gamma^{\prime}$ increases. For small damping $\left(\gamma^{\prime}<0.1\right)$, the frequency $\omega_{R}$ decreases with decreasing $\gamma^{\prime}$ for given values of $Q$. A very high-frequency band is visible in the spectrum of $\operatorname{Re}[\tilde{C}(\omega)]$ in Fig. 2 at moderate damping $\left(\gamma^{\prime}=1\right)$ due to the fast oscillations of the particles in the potential wells. For

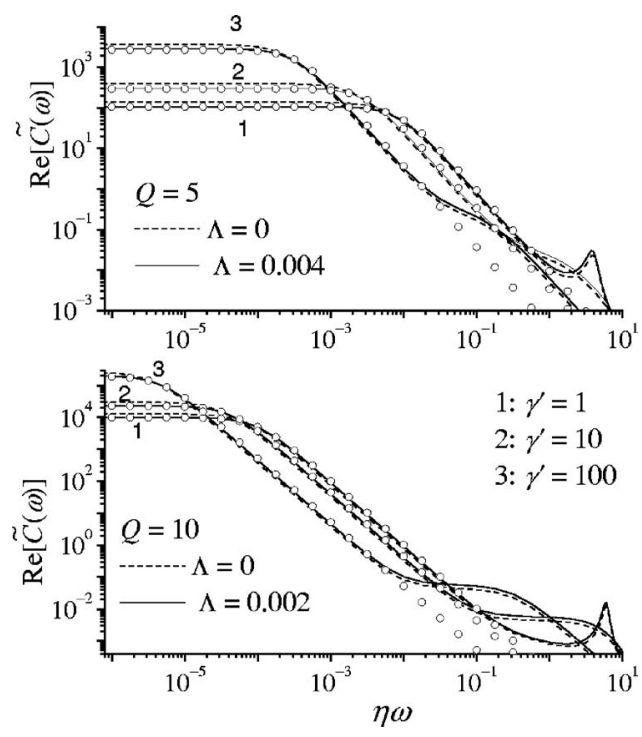

FIG. 2. The real part of the spectrum $\widetilde{C}(\omega)$ vs $\eta \omega$ for various values of $\gamma^{\prime}$, $\Lambda$, and $Q$. Key as in Fig. 1.
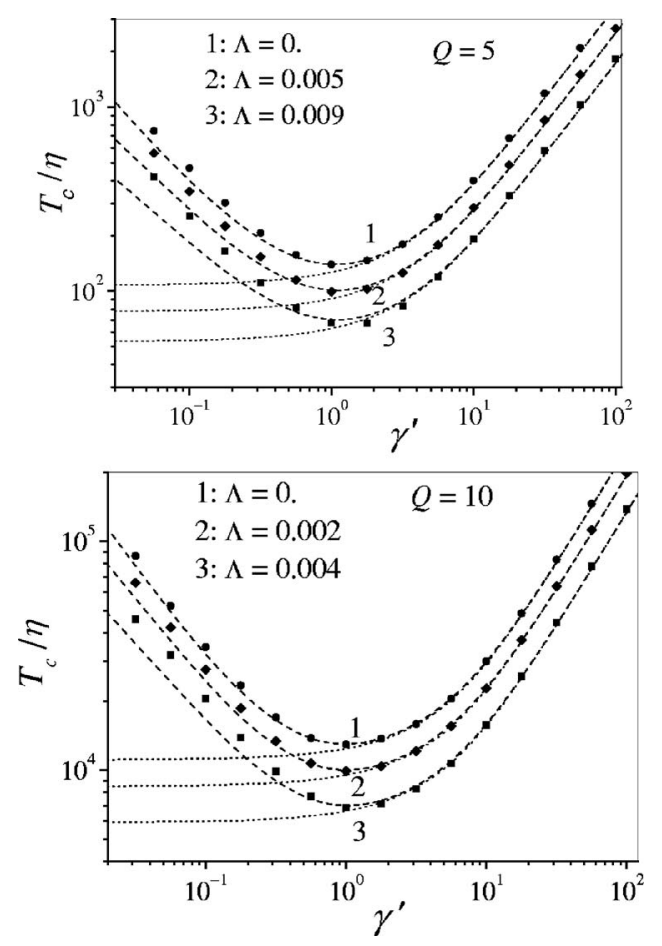

FIG. 3. Correlation time $T_{c} / \eta$ [Eq. (17), symbols] vs damping parameter $\gamma^{\prime}$ as compared with the universal Mel'nikov-Meshkov formula [Eq. (18), dashed lines] for high barriers $Q=5$ and various values of the quantum parameter $\Lambda$. The IHD relaxation times $\tau_{\mathrm{IHD}} / \eta=\left(\eta \Gamma_{\mathrm{IHD}}\right)^{-1}$ [Eq. (20), dotted lines 1] are also shown for comparison.

smaller damping, $\gamma^{\prime} \ll 1$, two sharp peaks appear in the highfrequency part of the spectra $\operatorname{Re}[\widetilde{C}(\omega)]$ just as in the classical case. $^{56}$ These peaks occur at the fundamental and second harmonic frequencies of the almost free periodic motion of the particle in the double well potential $V(x)$. We should remark that description of the quantum effects in the highfrequency response in the double well potential via the Wigner formalism applies only when the conditions $\eta \Delta E / \hbar \leqslant \gamma^{\prime}$ and $\Delta E / \hbar \leqslant \omega_{a}$ are satisfied. Here $\Delta E$ is the energy difference between the two lowest energy levels in the potential well and $\omega_{a} \sim \sqrt{2} Q^{3 / 4} / \eta$ is the characteristic frequency of the nonlinear oscillator. Under these conditions, the discrete spectral lines corresponding to the transitions between the energy levels in the wells are indistinguishable in the spectrum $\operatorname{Re}[\widetilde{C}(\omega)]$. For moderate barriers, the inequality $\eta \Delta E / \hbar \leqslant \gamma^{\prime}$ breaks down only for very small damping. Moreover, the classical limit holds if the conditions $\beta \Delta E \ll 1$ and $\eta \Delta E / \hbar \ll \gamma^{\prime}$ are satisfied.

The greatest relaxation time $\tau$ predicted by Melnikov's formula $\tau=\Gamma_{M}^{-1}$ [Eq. (18)] which we recall has been verified by computer simulation ${ }^{58}$ and the relaxation time $T_{c} / \eta$ from Eq. (17) calculated via matrix continued fractions are shown in Figs. 3 and 4 for barrier heights $Q=5$ and 10, respectively, as a function of the (dimensionless) damping parameter $\gamma^{\prime}$ characterizing the coupling to the heat bath. In relation to the matrix continued fraction calculations of the escape rate we recall that the (normalized) time $T_{c} / \eta$ being of exponential order accurately approximates the inverse escape rate for symmetric potentials for all significant barrier heights. We further remark that the inverse of the universal quantum rate 

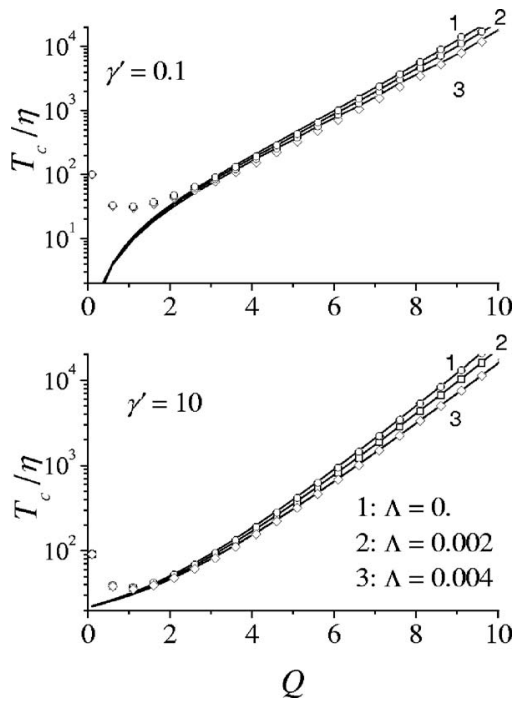

FIG. 4. The correlation time $T_{c} / \eta$ [matrix continued fraction solution, Eq (17), solid lines] vs the barrier parameter $Q$ for $\gamma^{\prime}=0.1$ (low damping) and $\gamma^{\prime}=10$ (high damping) and various values of the quantum parameter $\Lambda$ as compared with the universal Mel'nikov equation [Eq. (18)] (symbols).

provides a reasonably good approximation to $T_{c} / \eta$ for almost all $\gamma^{\prime}$ values with some deviation of the order of $20 \%$ for small friction $\gamma^{\prime}$ and relatively low barriers, e.g., $Q=5$ as $\Lambda$ increases. The agreement between the numerically calculated $\tau$ and the quantum rate result, however, improves as the barrier height increases (see Fig. 3). The results of the calculations suggest in applying quantum rate theory to relatively low barriers that the theory should be modified to incorporate finite-barrier effects as in the classical case as envisaged by Melnikov. ${ }^{63}$ In order to improve the accuracy of the universal turnover formula, Melnikov ${ }^{63}$ suggested a systematic way of accounting for finite-barrier corrections. Analysis of the translational Brownian motion in a cosine potential demonstrates that if such corrections are included, the accuracy of the universal formula is considerably improved. ${ }^{64,65}$ This method may also be applied to the present problem.

By way of further illustration we show in Fig. 4 the correlation time $T_{c} / \eta$ and the inverse of universal quantum rate $\left(\eta \Gamma_{M}\right)^{-1}$ as functions of barrier height $Q$ for large and small damping parameters $\gamma^{\prime}=10$ and 0.1 , respectively. Clearly, the correspondence between both results is very good for all relevant values of $Q$, e.g., $Q>3$. The discrepancy for $Q<2$ arises simply because the assumption of a high barrier $(Q \gg 1)$ is always used in the derivation of asymptotic escape rate formulas. The largest quantum effects obviously occur for the highest barriers as is obvious by inspection of Fig. 4. Moreover, they manifest themselves even for very small values of $\Lambda$.

To conclude, in this paper we have shown how quantum effects in the Brownian motion of a particle in a double well potential may be studied using a semiclassical master equation based on the extension of Wigner's phase space formulation of quantum mechanics to an open system. Our treatment allows one to use all the solution techniques previously developed for the classical Fokker-Planck equation ${ }^{1,2}$ rendering a transparent treatment of the quantum problem. More- over, our results are in agreement with those of quantum reaction rate theory which constitutes a benchmark solution verified by quantum mechanical simulations. ${ }^{58-60}$ The most significant manifestation of the quantum effects above the crossover temperature between tunneling and thermal activation appears to be in connection with the low-frequency relaxation via transitions across the potential barrier. In this frequency range, the relaxation process is accurately described by a single Lorentzian with relaxation time given by the inverse of Melnikov's universal quantum rate so providing a very simple picture of the quantum relaxation. Our matrix continued fraction solution is valid only for small values of the quantum parameter $\Lambda(\Lambda \ll 1)$ as in our perturbation procedure we have neglected all terms of the order of $\Lambda^{2}$ and higher. In order to improve the accuracy of matrix continued fraction calculations for larger values of $\Lambda$, additional terms of the order of $\Lambda^{2}$, etc. should be included in the master equation [Eq. (2)]. These higher order quantum correction terms to the master equation may be calculated, in principle, to any desired degree $r$ of $\hbar^{2 r} \cdot{ }^{24}$ Finally, we should remark in the context of our semiclassical solution that, hitherto, the quantum Brownian motion in a double well potential has usually been treated by means of numerical simulations (see, e.g., Refs. 22, 58-60, and 66). These methods in general allow one to understand quantum effects on diffusive transport properties, activated barrier crossing, etc. However, in spite of their great power they possess certain practical disadvantages, because the qualitative behavior of the simulated physical quantities is not always obvious from them. In general therefore one may expect that only a combined use of the complementary approaches of numerical simulation and the analytical methods described here is capable of yielding a comprehensive understanding of the quantum Brownian dynamics in a potential.

\section{ACKNOWLEDGMENT}

This publication has emanated from research conducted with the financial support of Science Foundation Ireland (Project No. 05/RFP/PHY/0070).

\section{APPENDIX A: MATRIX CONTINUED FRACTION SOLUTION OF EQ. (12)}

Noting that the recurrence Eq. (12) may be separated into two independent systems with $q+n$ even and odd, we introduce the column vectors

$$
\begin{aligned}
\mathbf{C}_{2 n-1}(t)= & \left(\begin{array}{c}
c_{2 n-2,1}(t) \\
c_{2 n-2,3}(t) \\
\vdots
\end{array}\right), \quad \mathbf{C}_{2 n}(t)=\left(\begin{array}{c}
c_{2 n-1,0}(t) \\
c_{2 n-1,2}(t) \\
\vdots
\end{array}\right) \\
& (n \geqslant 1) .
\end{aligned}
$$

Now Eq. (12) can be rearranged as the set of matrix recurrence equations for the one-sided Fourier transforms $\widetilde{\mathbf{C}}_{n}(\omega)$ $=\int_{0}^{\infty} \mathbf{C}_{n}(t) e^{-i \omega t} d t$, viz., 


$$
\begin{aligned}
\frac{d}{d t} \mathbf{C}_{n}\left(t^{\prime}\right)= & \mathbf{Q}_{n}^{-} \mathbf{C}_{n-1}\left(t^{\prime}\right)-\gamma^{\prime}(n-1) \mathbf{C}_{n}\left(t^{\prime}\right)+\mathbf{Q}_{n}^{+} \mathbf{C}_{n+1}\left(t^{\prime}\right) \\
& +\Lambda\left[\mathbf{q}_{n} \mathbf{C}_{n-2}\left(t^{\prime}\right)+\mathbf{r}_{n} \mathbf{C}_{n-3}\left(t^{\prime}\right)\right],
\end{aligned}
$$

where $\mathbf{r}_{n}, \mathbf{q}_{n}$, and $\mathbf{Q}_{n}^{ \pm}$are two-, three-, and four-diagonal matrices, respectively. Their matrix elements are given by

$$
\begin{aligned}
{\left[\mathbf{Q}_{2 n-\varepsilon}^{ \pm}\right]_{p, q}=} & \pm \sqrt{2 n-\varepsilon-\frac{1 \mp 1}{2}}\left(\delta_{p, q+2-\varepsilon} e_{2 p-5+\varepsilon}\right. \\
& +\delta_{p, q+1-\varepsilon} d_{2 p-3+\varepsilon}^{ \pm}+\delta_{p, q-\varepsilon} d_{2 p-2+\varepsilon}^{\mp} \\
& \left.+\delta_{p, q-1-\varepsilon} e_{2 p-2+\varepsilon}\right) \\
{\left[\mathbf{q}_{2 n-\varepsilon}\right]_{p, q}=} & \gamma^{\prime} \sqrt{(2 n-1-\varepsilon)(2 n-2-\varepsilon)}\left(\delta_{p, q+1} h_{2 p-4+\varepsilon}\right. \\
& \left.+\delta_{p, q} g_{2 p-2+\varepsilon}+\delta_{p, q-1} h_{2 p-2+\varepsilon}\right) \\
{\left[\mathbf{r}_{2 n-\varepsilon}\right]_{p, q}=} & \sqrt{(2 n-1-\varepsilon)(2 n-2-\varepsilon)(2 n-3-\varepsilon)} \\
& \times\left(f_{2 p-3+\varepsilon} \delta_{p, q+1-\varepsilon}+f_{2 p-2+\varepsilon} \delta_{p, q-\varepsilon}\right),
\end{aligned}
$$

where $\varepsilon=0,1$,

$$
\begin{aligned}
& d_{q}^{ \pm}=\frac{\alpha^{-3} B^{1 / 4}}{2} \sqrt{q+1}\left[3(q+1)-2 \alpha^{2} \sqrt{Q} \pm \alpha^{4}\right], \\
& e_{q}=\frac{B^{1 / 4} \alpha^{-3}}{2} \sqrt{(q+3)(q+2)(q+1)}, \\
& f_{q}=12 B^{3 / 4} \alpha^{-1} \sqrt{q+1}, \\
& h_{q}=12 \alpha^{-2} \sqrt{B(q+2)(q+1)}, \\
& g_{q}=4 \sqrt{B}\left[3 \alpha^{-2}(2 q+1)-2 \sqrt{Q}\right] .
\end{aligned}
$$

Next we use perturbation theory to find the solution of Eq. (A1) as

$$
\mathbf{C}_{n}\left(t^{\prime}\right)=\mathbf{C}_{n}^{0}\left(t^{\prime}\right)+\Lambda \mathbf{C}_{n}^{1}\left(t^{\prime}\right),
$$

treating $\Lambda$ as the customary small parameter. Substituting Eq. (A2) into Eq. (A1), we have in the zero order of perturbation theory the matrix three-term differential recurrence relation,

$$
\frac{d}{d t^{\prime}} \mathbf{C}_{n}^{0}\left(t^{\prime}\right)=\mathbf{Q}_{n}^{-} \mathbf{C}_{n-1}^{0}\left(t^{\prime}\right)-\gamma^{\prime}(n-1) \mathbf{C}_{n}^{0}\left(t^{\prime}\right)+\mathbf{Q}_{n}^{+} \mathbf{C}_{n+1}^{0}\left(t^{\prime}\right)
$$

and in the first order of perturbation theory the forced threeterm matrix differential recurrence relation

$$
\begin{aligned}
\frac{d}{d t^{\prime}} \mathbf{C}_{n}^{1}\left(t^{\prime}\right)= & \mathbf{Q}_{n}^{-} \mathbf{C}_{n-1}^{1}\left(t^{\prime}\right)-\gamma^{\prime}(n-1) \mathbf{C}_{n}^{1}\left(t^{\prime}\right) \\
& +\mathbf{Q}_{n}^{+} \mathbf{C}_{n+1}^{1}\left(t^{\prime}\right)+\mathbf{R}_{n}\left(t^{\prime}\right),
\end{aligned}
$$

where $\mathbf{R}_{n}\left(t^{\prime}\right)=\mathbf{q}_{n} \mathbf{C}_{n-2}^{0}\left(t^{\prime}\right)+\mathbf{r}_{n} \mathbf{C}_{n-3}^{0}\left(t^{\prime}\right)$.

By invoking the general method ${ }^{1,2}$ for solving three-term matrix recursion equations, we have the exact solution for the zero order spectrum $\widetilde{\mathbf{C}}_{n}^{0}(s)=\int_{0}^{\infty} \mathbf{C}_{n}^{0}(t) e^{-s t} d t$ in terms of a matrix continued fraction, viz.,

$$
\widetilde{\mathbf{C}}_{1}^{0}(s)=\boldsymbol{\Delta}_{1}(s) \mathbf{C}_{1}^{0}(0),
$$

$$
\widetilde{\mathbf{C}}_{n}^{0}(s)=\mathbf{S}_{n}^{-} \mathbf{C}_{n-1}^{0}(s)=\mathbf{S}_{n}^{-} \mathbf{S}_{n-1}^{-} \cdots \mathbf{S}_{2}^{-} \boldsymbol{\Delta}_{1}(s) \mathbf{C}_{1}^{0}(0),
$$

where $\mathbf{S}_{n}^{-}=\boldsymbol{\Delta}_{n}(s) \mathbf{Q}_{n}^{-}$and the matrix continued fraction $\boldsymbol{\Delta}_{n}(s)$ is defined by the recurrence equation,

$$
\boldsymbol{\Delta}_{n}(s)=\left\{\left[s+\gamma^{\prime}(n-1)\right] \mathbf{I}-\mathbf{Q}_{n}^{+} \boldsymbol{\Delta}_{n+1}(s) \mathbf{Q}_{n+1}^{-}\right\}^{-1} .
$$

In a similar manner, we also have the exact solution for the first order spectrum $\widetilde{\mathbf{C}}_{1}^{1}(s)$ in terms of a matrix continued fraction, viz.,

$$
\widetilde{\mathbf{C}}_{1}^{1}(s)=\boldsymbol{\Delta}_{1}(s)\left\{\mathbf{C}_{1}^{1}(0)+\mathbf{S}_{2}^{+} \mathbf{S}_{3}^{+}\left[\mathbf{C}_{3}^{1}(0)+\mathbf{F}\right]\right\},
$$

where $\mathbf{S}_{n}^{+}=\mathbf{Q}_{n-1}^{+} \boldsymbol{\Delta}_{n}(s)$ and

$$
\begin{aligned}
\mathbf{F}= & \left\{\mathbf{q}_{3}+\mathbf{S}_{4}^{+}\left[\mathbf{q}_{4} \mathbf{S}_{2}^{-}+\mathbf{r}_{4}\right.\right. \\
& \left.\left.+\sum_{n=5}^{\infty} \mathbf{S}_{5}^{+} \cdots \mathbf{S}_{n}^{+}\left(\mathbf{q}_{n} \mathbf{S}_{n-2}^{-}+\mathbf{r}_{n}\right) \mathbf{S}_{n-3}^{-} \cdots \mathbf{S}_{2}^{-}\right]\right\} \boldsymbol{\Delta}_{1}(s) \mathbf{C}_{1}^{0}(0) .
\end{aligned}
$$

Here we have noted that in the first order of perturbation theory, the only nonzero initial conditions $\mathbf{C}_{1}^{0}(0), \mathbf{C}_{1}^{1}(0)$, and $\mathbf{C}_{3}^{1}(0)$ [as dictated by the Wigner distribution equation, Eqs. (6) and (10)]. Thus the only nonzero initial conditions $c_{m, 2 p+1}(0)=c_{m, 2 p+1}^{0}(0)+\Lambda c_{m, 2 p+1}^{1}(0) \quad$ with $c_{m, 2 p+1}^{k}(0) \quad(m=0,2$ and $k=0,1)$ given by

$$
c_{m, 2 p+1}^{k}(0)=\frac{\int_{-\infty}^{\infty} \xi F_{m}^{k}(\xi) H_{2 p+1}(\alpha \xi) e^{-\left(\alpha^{2} \xi^{2}-2 \sqrt{Q} \xi^{2}+\xi^{4}\right) / 2} d \xi}{Z_{\mathrm{cl}} \sqrt{2^{2 p+1}(2 p+1) !}},
$$

where

$$
\begin{aligned}
& F_{0}^{0}(\xi)=1 / \sqrt{B}, \quad F_{2}^{0}(\xi)=0, \\
& F_{0}^{1}(\xi)=16\left[\xi^{2}\left(-\sqrt{Q}+\xi^{2}\right)^{2}+\sqrt{Q}-2 \xi^{2}\right]-\frac{Z_{1}}{Z_{\mathrm{cl}} \sqrt{B}}, \\
& F_{2}^{1}(\xi)=4 \sqrt{2}\left(3 \xi^{2}-\sqrt{Q}\right), \\
& Z_{\mathrm{cl}}=\int_{-\infty}^{\infty} e^{-V\left(x^{\prime}\right)} d x^{\prime}=\sqrt{\pi}(2 B)^{-1 / 4} D_{-1 / 2}(-\sqrt{2 Q}) e^{Q / 2},
\end{aligned}
$$

and

$$
\begin{aligned}
\frac{Z_{1}}{\sqrt{B} Z_{\mathrm{cl}}}= & \frac{1}{\sqrt{B} Z_{\mathrm{cl}}} \int_{-\infty}^{\infty}\left[V^{\prime}\left(x^{\prime}\right)^{2}-2 V^{\prime \prime}\left(x^{\prime}\right)\right] e^{-V\left(x^{\prime}\right)} d x^{\prime} \\
= & -\sqrt{8}\left[D_{1 / 2}(-\sqrt{2 Q})\right. \\
& \left.+D_{-3 / 2}(-\sqrt{2 Q})\right] / D_{-1 / 2}(-\sqrt{2 Q}) .
\end{aligned}
$$

\section{APPENDIX B: THE DERIVATION OF $R(\lambda, y)$ FOR THE DOUBLE WELL POTENTIAL}

To obtain $R(\lambda, y)$ occurring in the integral of the depopulation factor $A^{\prime}(\Delta, y)$, Eq. (25), Melnikov ${ }^{49}$ calculated the transition probability $g\left(\varepsilon, \varepsilon^{\prime}\right)$ from the state $\varepsilon^{\prime}$ to the state $\varepsilon$ using perturbation theory. The expression for $g\left(\varepsilon-\varepsilon^{\prime}\right)$ becomes $^{49}$ 


$$
\begin{aligned}
g\left(\varepsilon-\varepsilon^{\prime}\right)= & e^{-\int_{-\infty}^{\infty} w(\varepsilon) d \varepsilon}\left\{w\left(\varepsilon-\varepsilon^{\prime}\right)\right. \\
& \left.+\frac{1}{2} \int_{-\infty}^{\infty} w\left(\varepsilon-\varepsilon^{\prime \prime}\right) w\left(\varepsilon^{\prime \prime}-\varepsilon^{\prime}\right) d \varepsilon^{\prime \prime}+\cdots\right\},
\end{aligned}
$$

where $w$ is the quantum transition probability in first order perturbation theory,

$w\left(\varepsilon-\varepsilon^{\prime}\right)=\frac{2 \pi m}{\hbar}\left|\left\langle\varepsilon|w| \varepsilon^{\prime}\right\rangle\right|^{2} \gamma\left(\varepsilon-\varepsilon^{\prime}\right)\left\{\operatorname{coth}\left[\beta\left(\varepsilon-\varepsilon^{\prime}\right) / 2\right]-1\right\}$.

Taking the Fourier transform, $f(\lambda)=\int_{-\infty}^{\infty} f(\varepsilon) e^{i \lambda \beta \varepsilon} d \varepsilon$, of Eq. (B1) and performing the summation, we have

$$
g(\lambda)=e^{w(\lambda)-w(0)}=e^{-(\beta \gamma \delta) R(\lambda+i / 2, \beta \hbar \omega / 2)} .
$$

Now in the semiclassical approximation the matrix elements $\left\langle\varepsilon|x(t)| \varepsilon^{\prime}\right\rangle$ pertaining to a quantum transition from the state $\varepsilon^{\prime}$ to the state $\varepsilon$ can be expressed in terms of the Fourier components of the classical trajectory $x(t)$,

$$
\left\langle\varepsilon|\hat{x}(t)| \varepsilon^{\prime}\right\rangle=\frac{1}{2 \pi \hbar} \int_{-\infty}^{\infty} x(t) e^{i\left(\varepsilon-\varepsilon^{\prime}\right) t / \hbar} d t
$$

We need the classical trajectory $x(t)$ corresponding to zero energy, i.e., the saddle energy trajectory $\varepsilon=0$. It is defined by the implicit relation

$$
t(x)= \pm \int_{x_{1}}^{x} \frac{d x^{\prime}}{\sqrt{-2 V\left(x^{\prime}\right) / m}}
$$

where $x_{1}$ is the classical turning point at $t=0$ and the signs + and - correspond to positive and negative velocities of the particle, respectively. The particle starts from $x=0$ at $t=-\infty$ and returns to this point as $t \rightarrow \infty$. For the particular case of the potential, Eq. (1), we have

$$
t(x)=\frac{1}{\omega} \int_{x_{1}}^{x} \frac{d x^{\prime}}{x^{\prime} \sqrt{1-x^{\prime 2} / x_{1}^{2}}}=-\frac{i}{\omega} \arccos \left(x / x_{1}\right)
$$

or

$$
x(t)=x_{1} / \cosh (\omega t)
$$

where $\omega=\sqrt{-a / m}$ and $x_{1}=\omega \sqrt{2 m / b}$. The matrix elements $\left\langle\varepsilon|x(t)| \varepsilon^{\prime}\right\rangle$ for the double well potential are then given by

$$
\begin{aligned}
\left\langle\varepsilon|\hat{x}(t)| \varepsilon^{\prime}\right\rangle & =\frac{x_{1}}{2 \pi \hbar} \int_{-\infty}^{\infty} \frac{e^{i\left(\varepsilon-\varepsilon^{\prime}\right) t / \hbar} d t}{\cosh (\omega t)} \\
& =\frac{x_{1}}{2 \hbar \omega \cosh \left[\pi\left(\varepsilon-\varepsilon^{\prime}\right) / 2 \hbar \omega\right]} .
\end{aligned}
$$

The quantum transition probability $w(\varepsilon)$ and its Fourier transform, namely, $w(\lambda-i / 2)-w(0)$, are then given by, respectively,

$$
w(\varepsilon)=\frac{\pi m \gamma x_{1}^{2} \varepsilon(\operatorname{coth}[\beta \varepsilon / 2]-1)}{2 \hbar^{3} \omega^{2} \cosh ^{2}[\pi \varepsilon / 2 \hbar \omega]},
$$

and

$$
\begin{aligned}
w(\lambda & -i / 2)-w(0) \\
& =\int_{-\infty}^{\infty} w(\varepsilon)\left(e^{i(\lambda-i / 2) \beta \varepsilon}-1\right) d \varepsilon \\
& =\beta \gamma \frac{2 \pi \omega m x_{1}^{2}}{8} \int_{-\infty}^{\infty} \frac{x(\cosh y x-\cos 2 \lambda y x)}{y \sinh y x \cosh ^{2}(\pi x / 2)} d x \\
& =\beta \gamma \operatorname{SR}(\lambda, y) .
\end{aligned}
$$

Here $S=2 \int_{0}^{x_{1}} \sqrt{-2 m V(x)} d x=2 \omega m x_{1}^{2} / 3, y=\beta \hbar \omega / 2, x=\beta \varepsilon / 2 y$, and

$$
R(\lambda, y)=\frac{3 \pi}{8 y} \int_{-\infty}^{\infty} \frac{x(\cosh y x-\cos 2 \lambda y x)}{\sinh y x \cosh ^{2}(\pi x / 2)} d x .
$$

Noting that

$$
\lim _{\hbar \rightarrow 0} \frac{\cosh y x-\cos 2 \lambda y x}{y \sinh (y x)}=\frac{x}{2}\left(1+4 \lambda^{2}\right),
$$

we have in the classical limit

$$
\lim _{\hbar \rightarrow 0} R(\lambda, y)=\frac{3 \pi\left(\lambda^{2}+1 / 4\right)}{4} \int_{-\infty}^{\infty} \frac{x^{2} d x}{\cosh ^{2}(\pi x / 2)}=\lambda^{2}+1 / 4 .
$$

${ }^{1}$ H. Risken, The Fokker-Planck Equation, 2nd ed. (Springer, Berlin, 1989).

${ }^{2}$ W. T. Coffey, Yu. P. Kalmykov, and J. T. Waldron, The Langevin Equation, 2nd ed. (World Scientific, Singapore, 2004).

${ }^{3}$ P. Hänggi and G. L. Ingold, Chaos 15, 026105 (2005).

${ }^{4}$ P. Hänggi, P. Talkner, and M. Berkovec, Rev. Mod. Phys. 62, 251 (1990).

${ }^{5}$ D. Kohen and D. J. Tannor, Adv. Chem. Phys. 111, 219 (1994).

${ }^{6}$ E. P. Wigner, Phys. Rev. 40, 749 (1932).

${ }^{7}$ S. R. de Groot and L. G. Suttorp, Foundations of Electrodynamics (North-Holland, Amsterdam, 1972), Chap. VI-VII.

${ }^{8}$ M. Hillery, R. F. O'Connell, M. O. Scully, and E. P. Wigner, Phys. Rep. 106, 121 (1984).

${ }^{9}$ V. I. Tatarskii, Usp. Fiz. Nauk 139, 587 (1983) [Sov. Phys. Usp. 26, 311 (1983)].

${ }^{10}$ H. W. Lee, Phys. Rep. 259, 147 (1995).

${ }^{11}$ W. P. Schleich, Quantum Optics in Phase Space (Wiley-VCH, Berlin, 2001).

${ }^{12}$ R. Kapral, Annu. Rev. Phys. Chem. 57, 129 (2006).

${ }^{13}$ G. S. Agarwal, Phys. Rev. A 4, 739 (1971).

${ }^{14}$ H. Dekker, Phys. Rev. A 16, 2116 (1977).

${ }^{15}$ J. J. Halliwell and T. Yu, Phys. Rev. D 53, 2012 (1996).

${ }^{16}$ R. Karrlein and H. Grabert, Phys. Rev. E 55, 153 (1997).

${ }^{17}$ U. Weiss, Quantum Dissipative Systems (World Scientific, Singapore, 1999).

${ }^{18}$ G. W. Ford and R. F. O'Connell, Phys. Rev. D 64, 105020 (2001).

${ }^{19}$ H. Grabert, Chem. Phys. 322, 160 (2006).

${ }^{20}$ H. T. Davis, K. Hiroike, and S. A. Rice, J. Chem. Phys. 43, 2633 (1965).

${ }^{21}$ Y. Tanimura and P. G. Wolynes, J. Chem. Phys. 86, 8485 (1992).

${ }^{22}$ S. Zhang and E. Pollak, J. Chem. Phys. 118, 4357 (2003).

${ }^{23}$ J. L. García-Palacios, Europhys. Lett. 65, 735 (2004); J. L. GarcíaPalacios and D. Zueco, J. Phys. A 37, 10735 (2004).

${ }^{24}$ W. T. Coffey, Yu. P. Kalmykov, S. V. Titov, and B. P. Mulligan, Europhys. Lett. 77, 20011 (2007); J. Phys. A: Math. Theor. 40, F91 (2007).

${ }^{25}$ W. T. Coffey, Yu. P. Kalmykov, S. V. Titov, and B. P. Mulligan, Phys. Chem. Chem. Phys. 9, 3361 (2007).

${ }^{26}$ W. T. Coffey, Yu. P. Kalmykov, S. V. Titov, and B. P. Mulligan, Phys. Rev. E 75, 041117 (2007).

${ }^{27}$ D. Chandler, J. Chem. Phys. 68, 2959 (1978).

${ }^{28}$ B. J. Berne, J. L. Skinner, and P. G. Wolynes, J. Chem. Phys. 73, 4314 (1980).

${ }^{29}$ D. L. Hasha, T. Eguchi, and J. Jonas, J. Chem. Phys. 75, 1571 (1981); J. Am. Chem. Soc. 104, 2290 (1982).

${ }^{30}$ D. K. Garrity and J. L. Skinner, Chem. Phys. Lett. 95, 46 (1983).

${ }^{31}$ B. Carmeli and A. Nitzan, J. Chem. Phys. 80, 3596 (1984).

${ }^{32}$ H. A. Kramers, Physica (Amsterdam) 7, 284 (1940). 
${ }^{33}$ H. C. Brinkman, Physica (Amsterdam) 22, 29 (1956); 22, 149 (1956).

${ }^{34}$ C. Blomberg, Physica A 86, 49 (1977); 86, 67 (1977).

${ }^{35}$ P. B. Visscher, Phys. Rev. B 14, 347 (1976).

${ }^{36}$ J. L. Skinner and P. G. Wolynes, J. Chem. Phys. 69, 2143 (1978); 72 , 4913 (1980)

${ }^{37}$ R. S. Larson and M. D. Kostin, J. Chem. Phys. 69, 4821 (1978); 72 , $1392(1980)$

${ }^{38}$ S. C. Northrup and J. T. Hynes, J. Chem. Phys. 69, 5246 (1978); 69, 5261 (1978); R. F. Grote and J. T. Hynes, ibid. 73, 2700 (1980); 73, 2715 (1980).

${ }^{39}$ M. Mangel, J. Chem. Phys. 72, 6606 (1980).

${ }^{40}$ K. Schulten, Z. Schulten, and A. Szabo, J. Chem. Phys. 74, 4426 (1981).

${ }^{41}$ M. Bixon and R. Zwanzig, J. Stat. Phys. 3, 245 (1971).

${ }^{42}$ M. I. Dykman, S. M. Soskin, and M. A. Krivoglaz, Physica A 133, 53 (1985).

${ }^{43}$ P. Hänggi, Phys. Lett. 78A, 304 (1980).

${ }^{44}$ J. A. Krumhansl and J. R. Schriefier, Phys. Rev. B 11, 3535 (1975).

${ }^{45}$ J. D. Bao and Y. Z. Zhuo, Phys. Rev. C 67, 064606 (2003).

${ }^{46}$ V. M. Kolomietz, S. V. Radionov, and S. Shlomo, Phys. Rev. C 64 054302 (2001).

${ }^{47}$ M. Grifoni, L. Hartmann, S. Berchtold, and P. Hänggi, Phys. Rev. E 53, 5890 (1996).

${ }^{48}$ L. Gammaitoni, P. Hänggi, P. Jung, and F. Marchesoni, Rev. Mod. Phys. 70, 223 (1998)

${ }^{49}$ V. I. Mel'nikov, Phys. Rep. 209, 1 (1991); Physica A 130, 606 (1985).
${ }^{50}$ V. I. Mel'nikov and S. V. Meshkov, J. Chem. Phys. 85, 1018 (1986).

${ }^{51}$ H. Grabert, Phys. Rev. Lett. 61, 1683 (1988).

${ }^{52}$ E. Pollak, H. Grabert, and P. Hänggi, J. Chem. Phys. 91, 4073 (1989).

${ }^{53}$ I. Rips and E. Pollak, Phys. Rev. A 41, 5366 (1990).

${ }^{54}$ K. Voigtlaender and H. Risken, J. Stat. Phys. 40, 397 (1985); Chem. Phys. Lett. 105, 506 (1984).

${ }^{55}$ A. Perico, R. Pratolongo, K. F. Freed, R. W. Pastor, and A. Szabo, J. Chem. Phys. 98, 564 (1993).

${ }^{56}$ Yu. P. Kalmykov, W. T. Coffey, and S. V. Titov, J. Chem. Phys. 124, 024107 (2006).

${ }^{57}$ Yu. P. Kalmykov, W. T. Coffey, and S. V. Titov, Physica A 377, 412 (2007).

${ }^{58}$ M. Topaler and N. Makri, J. Chem. Phys. 101, 7500 (1994).

${ }^{59}$ N. Makri, J. Math. Phys. 36, 2430 (1995).

${ }^{60}$ D. Barik, B. C. Bag, and D. S. Ray, J. Chem. Phys. 119, 12973 (2003).

${ }^{61}$ Handbook of Mathematical Functions, edited by M. Abramowitz and I. Stegun (Dover, New York, 1964).

${ }^{62}$ R. Kubo, J. Phys. Soc. Jpn. 12, 570 (1957); R. Kubo, M. Toda, and N. Hashitsume, Statistical Physics II. Nonequilibrium Statistical Mechanics, 2nd ed. (Springer, Berlin, 1991).

${ }^{63}$ V. I. Mel'nikov, Phys. Rev. E 48, 3271 (1993); 50, 627 (1994).

${ }^{64}$ P. Talkner and E. Pollak, Phys. Rev. E 47, R21 (1993).

${ }^{65}$ R. Ferrando, R. Spadacini, G. E. Tommei, and V. I. Mel'nikov, Phys. Rev. E 51, R1645 (1995).

${ }^{66}$ J. T. Stockburger and C. H. Mak, J. Chem. Phys. 110, 4983 (1999). 